\title{
One nerve suffices: A clinically guided nerve ultrasound protocol for the differentiation of multifocal motor neuropathy (MMN) and amyotrophic lateral sclerosis (ALS)
}

\author{
Kai F. Loewenbrück ${ }^{1,2} \cdot$ Robin Werner $^{1} \cdot$ René Günther $^{1,2} \cdot$ Markus Dittrich $^{1,3} \cdot$ Robert Klingenberger $^{1}$. \\ Heinz Reichmann ${ }^{1} \cdot$ Alexander Storch ${ }^{4,5} \cdot$ Andreas Hermann $^{5,6}$
}

Received: 12 September 2020 / Revised: 14 November 2020 / Accepted: 19 November 2020 / Published online: 23 December 2020

(c) The Author(s) 2020

\begin{abstract}
Objective To investigate diagnostic accuracy of a nerve ultrasound (US) protocol that is individualized to a patient's clinical deficits for the differentiation of amyotrophic lateral sclerosis with predominant lower motoneuron disease (ALS/LMND) and multifocal motor neuropathy (MMN).

Methods Single-center, prospective, examiner-blinded, diagnostic study in two cohorts. Cohort I (model development): Convenience sample of subjects with ALS/LMND or MMN according to revised El-Escorial or EFNS guidelines. Cohort II (model validation): Consecutively recruited treatment-naïve subjects with suspected diagnosis of ALS/LMND or MMN. Cutoffs for 28 different US values were determined by Receiver Operating Curve (ROC) in cohort I. Area Under The Curve (AUC) of US was compared to nerve conduction studies (NCS). Diagnostic accuracy of US protocols, individualized according to clinical deficits, was compared to former rigid non-individualized protocols and to random examination site selection in cohort II.

Results 48 patients were recruited. In cohort I (28 patients), US had higher ROC AUCs than NCS, US 0.82 (0.12) (mean (standard deviation)), NCS (compound muscle action potential (CMAP) 0.60 (0.09), $p<.001$; two-sided $t$-test).

US models based on the nerve innervating the clinically most affected muscles had higher correct classification rates (CCRs, $93 \%)$ in cohort II than former rigid protocols $(85 \%$ and $80 \%)$, or models with random measurement site selection ( $66 \%$ and $80 \%)$.

Conclusions Clinically guided US protocols for differentiation of ALS/LMND from MMN increase diagnostic accuracy when compared to clinically unguided protocols. They also require less measurements sites to achieve this accuracy.
\end{abstract}

Keywords Nerve ultrasound $\cdot$ Als $\cdot$ Mmn $\cdot$ Diagnosis $\cdot$ Nerve conduction studies

\section{Introduction}

Supplementary Information The online version contains supplementary material available at https://doi.org/10.1007/s0041 5-020-10323-6.

Kai F. Loewenbrück

kai.loewenbrueck@uniklinikum-dresden.de

1 Department of Neurology, Technische Universität Dresden, Fetscherstrasse 74, 01307 Dresden, Germany

2 German Center for Neurodegenerative Diseases (DZNE), 01307 Dresden, Germany

3 Department of Neurology, Elblandkliniken, 01662 Meissen, Germany
The differential diagnosis between amyotrophic lateral sclerosis with leading lower motoneuron disease presentation (ALS/LMND) and multifocal motor neuropathy

4 Department of Neurology, University of Rostock, 18147 Rostock, Germany

5 German Center for Neurodegenerative Diseases (DZNE) Rostock/Greifswald, 18147 Rostock, Germany

6 Department of Neurology, Translational Neurodegeneration Section "Albrecht Kossel”, University of Rostock, 18147 Rostock, Germany 
$(\mathrm{MMN})$ is of high prognostic and therapeutic importance while clinically sometimes demanding. Whereas ALS/ LMND represents a fatal condition with no relevant disease-modifying treatment options, immune-modulating therapies are effective in MMN and increase strength and functional outcome [1].

Several studies have illustrated the value of nerve ultrasound (US) in this differential diagnostic question [2-4], reaching up to $100 \%$ sensitivity and $92 \%$ specificity for MMN in external validation study groups. The underlying diagnostic contrast is driven by a slight decrease in nerve cross-sectional area (CSA) in ALS/LMND on the one side [5], possibly due to accompanying axonal loss, and by a pronounced increase in CSA in MMN as a chronic inflammatory neuropathy on the other side [6].

Further studies support the clinical relevance of US assessments in patients with ALS/LMND or MMN. US measurements correlate with clinical scores like the Medical Research Council Sum Score (MRC-SS) and the Overall Disability Sum Score (ODSS) in MNN or with the MRC-SS and the revised ALS Functional Rating Scale (ALSFRS-R) in ALS/LMND [4]. In ALS, a decrease in CSA is limited to subtypes with lower motor neuron involvement and is not existent in primary lateral sclerosis (PLS) as a subtype without such involvement [7]. In addition, in ALS CSA correlates with cerebrospinal fluid (CSF) progranulin levels as a biochemical marker for axonal damage [8]. In MMN, CSA enlargements correlate with intraindividual longitudinal changes in clinical deficits and might therefore be suitable for the monitoring of therapeutic effects [6].

The above-mentioned diagnostic US models to differentiate MMN from ALS/LMND are based on standard examination protocols including 8 [4] or 10 [2] US measurements sites in different nerves, irrespective of where an individual patient shows his or her strongest clinical deficits. Such rigid and clinically unguided examination protocols are not only relatively laborious, but could have compromised diagnostic accuracy. The nerves included in the respective protocol are not necessarily the ones the strongest affected in an individual patient. Furthermore, repetitive measurements increase the risk of false-positive results.

The current prospective, monocenter, rater-blinded, disease-controlled diagnostic study tested the hypothesis that a clinically guided US examination of the clinically most strongly affected nerve only has equal or superior diagnostic power and is more efficient than the abovementioned rigid and clinically unguided examination protocols for the differentiation of MMN from ALS/LMND. Former diagnostic US studies have been criticized for a potential spectrum bias due to study groups not representative of the diagnostic target population [9]. To address this, the hypothesis of the current study was tested in a prospectively recruited patient cohort without an established diagnosis of ALS/LMND or MMN, admitted for differential diagnosis of subacute acquired peripheral pure motor deficits of unknown etiology.

\section{Subjects and methods}

\section{Study design, subjects and group assignment}

This single-center, prospective, examiner-blinded cross-sectional diagnostic study was performed at the Department of Neurology, Technische Universität Dresden, Saxony, Germany. Two study cohorts were recruited between May 11th, 2017 and July 17th, 2018 (see Online Resource Figs 1 and 2 for study flow diagrams).

Cohort I consisted of patients with clinically established diagnoses and was used to develop diagnostic models and define US cutoff values. Cohort II consisted of patients admitted for the differential diagnosis of either ALS/LMND or MMN and served as an external validation group for the models.

Cohort I: Cohort I was conveniently recruited at our specialized outpatient clinic for patients with ALS and inflammatory neuropathies. Inclusion criteria were the diagnosis of ALS/LMND or MMN. For ALS, minimal diagnostic criterion was possible ALS according to revised El-Escorial criteria in combination with predominant LMN involvement [10]. Predominant LMN involvement was defined as presence of paresis without clinical or electrophysiological sings of upper motor neuron (UMN) involvement, such as increased muscle tone, spasticity, presence of pathological reflexes or increased central motor latency in magnetic evoked potentials. For MMN, minimal diagnostic criterion was a possible MMN according to the European Federation of Neurosciences/Peripheral Nerve Society (EFNS/PNS) guidelines [11].

Cohort II: Cohort II was a consecutive sample recruited from all patients admitted between May 11th, 2017 and July 17th, 2018 with a suspected diagnosis of ALS/LMND or MMN. For study eligibility, this differential diagnostic focus had to be reconfirmed by the attending senior physician during initial clinical ward rounds.

Exclusion criterion was inability to follow the study protocol (e.g., due to advanced impairment or severe dyspnea). All patients provided written informed consent. The study was approved by the institutional review board.

\section{Procedures}

Basic demographic data were recorded (age, sex, disease duration and time since diagnosis in months). For clinical 
signs and symptoms, the following scales were employed: Medical Research Council Sum Score (MRC-SS) [12], Modified Rankin Scale (mRS) [13], EuroQol (EQ-5D-5L) [14] revised ALS Functional Rating Scale (ALSFRS-R, ALS/ LMND only) [15], Overall Disability Sum Score (ODSS, MMN only) [13], Rasch-built Overall Disability Scale for Multifocal motor neuropathy (MMN-RODS, MMN only) [14]. Grip force was assessed with a dynamometer (Jamar hydraulic handgrip dynamometer, Lafayette Instrument Inc., Loughborough, UK).

Motor and sensory NCS (motor and sensory nerve conduction velocities (CVs), compound muscle action potentials (CMAPs)) of median, ulnar, radial, tibial, peroneal and sural nerves and f-responses of the median, ulnar and tibial nerves were obtained on a Keypoint electrophysiological system, Software Version 3.5 (Medtronic, Meerbusch, Germany) by experienced examiners (C.L., J.D.) blinded to group assignment. In addition to the measurement values, the presence of motor conduction blocks (defined as a reduction of $>50 \%$ in amplitude or area under the curve (AUC)) was determined. Patients were examined unilaterally on the clinically more affected side.

Both transverse and longitudinal US scans were obtained in 12 nerves/nerve roots at 14 different sites to record CSA and diameter (nerve/nerve roots examined: $\mathrm{C} 5,6,7$, superior trunk, vagal, radial, ulnar, median, sciatic, peroneal, tibial and sural nerve). Three images were taken at each measurement site and the mean calculated. Measurement sites were chosen according to a highly standardized and reproducible study protocol proposed by Zaidman and co-workers [16]. US machines used were: Aplio MX, linear transducer, 8-18 MHz (Toshiba, Neuss, Germany) and MyLab Five, linear transducer, 10-18 MHz (Esaote Biomedica, Cologne, Germany). Examinations were performed by two experienced investigators (R.W., K.F.L.), blinded to group assignment.

\section{Statistical analysis and diagnostic models}

Statistical significance was defined by $p<0.05$. If not otherwise stated, numbers given are mean and standard deviation (SD), and the $p$ value derived from a two-sided $t$-test. Normal distribution of continuous data was tested with Kolmogorov-Smirnov test. If data were normally distributed, two-sided $t$-tests were used for all two-group comparisons, otherwise a univariate ANOVA with a post hoc two-sided $t$-test. If data were not normally distributed or ordinal, a Mann-Whitney- $U$-test or a Kruskal-Wallis test with a post-hoc Mann-Whitney- $U$-test was used. All post hoc tests were Bonferroni-adjusted for $\alpha$-inflation. For categorical data, Fisher's exact test was used, in case of three groups with a Freeman-Halton extension. Correlations were assessed by Pearson's correlation coefficient.
Receiver operating curve (ROC) analysis was performed to compare the area under the curve (AUC) for all single measurements obtained by US and NCS and to obtain cutoff values for all US measurements. Cutoff values were chosen from the respective ROC analysis in cohort I as defined by the highest Youden-index, or a minimum specificity of $\geq 80 \%, \geq 90 \%, \geq 95 \%$ or $100 \%$.

The nerve/nerve root with the most heavily affected downstream muscle functions was determined by MRCSS-based clinical testing of single muscle functions that could be unequivocally assigned to a single nerve; in case of that several muscle functions could be attributed to one nerve, the mean was considered (for muscle-nerve assignment, see Table 1). Functions that could be assigned to more than one nerve were not considered. Nerve roots naturally shared single muscle functions with peripheral nerves and were differentiated as far as possible based on single muscle functions (Table 1).

In case of that the above-mentioned clinical testing procedure resulted into two or more nerves/nerve roots being affected to the same extent, the following four strategies were applied to define the single nerve/nerve root to be included into an individualized US testing protocol. All strategies were systematically compared for their diagnostic performance (for details, see Fig. 2):

(1) In case of that the heaviest single muscle deficit could either be attributed to a consecutive proximal or distal nervous structure (e.g., C5 root vs. superior trunk; C6 root vs. superior trunk; $\mathrm{C} 7$ root vs. radial nerve; sciatic nerve vs. peroneal nerve or sciatic nerve vs. tibial nerve): Systematic comparison of protocols that either included the more proximal or the more distal structure (more proximal: models 1, 2, 5 \& 6 vs. more distal: models $3,4,7$ \& 8, Fig. 2).

(2) Systematic comparison of protocols that did or did not consider nerve roots in the examination site determination process (with cervical roots: models $2,4,6 \& 8$ vs. without cervical roots: models $1,3,5 \& 7$, Fig. 2).

(3) In case of that equal and heaviest muscle deficits were present in two distinct nonconsecutive nervous structures: Decision for the nerve/nerve root easier to assess by US according to the following order of increasing difficulty: arm < leg < neck; ulnar nerve $<$ median nerve $<$ radial nerve; peroneal nerve $<$ tibial nerve; $\mathrm{C} 5$ root $<\mathrm{C} 6$ root $<\mathrm{C} 7$ root; superior trunk $<\mathrm{C} 7$ root (applied to all models 1-8, Fig. 2).

(4) Comparison of models that consider both CSA and diameter or CSA only (Both CSA and diameter: models $1,2,3, \& 4$ vs. CSA only: models $5,6,7 \& 8$ ).

The application of these four selection rules resulted in eight different models (Fig. 2). Based on the cutoff values 
determined by ROC analysis in cohort I (Table 2), the diagnostic performance of the eight different models was systematically assessed in both cohorts I \& II (Fig. 3). In nerves where two measurement sites were assessed (median and ulnar nerve), both sites were considered and scored as pathological if either one of the two exceeded the respective cutoffs. In addition, the diagnostic performance of these eight different models guided by the distribution of clinical deficits was compared to formerly published clinically-unguided protocols $[4,17]$ (models 11 \& 12), and to random clinically unguided examination site selection (models $9 \& 10$ ) (Fig. 3).

All analyses were performed with SPSS Version 25 (IBM, Ehningen, Germany).

\section{Results}

48 patients were included, of which 28 allocated to cohort I for diagnostic model development and 20 to cohort II for subsequent external model testing.

Cohort I was conveniently recruited and comprised 20 patients with ALS/LMND and 8 with MMN. For external testing in a study cohort representative of a realistic diagnostic target population, cohort II consisted of 20 patients with a clinically suspected diagnosis of ALS/ LMND or MMN, consecutively recruited from all 601 patients admitted to the general neurology ward of the study center between May 11th, 2017 and July 17th, 2018 (details in Table 3). After comprehensive routine diagnostic procedures (US as index test not considered), the 20 patients of cohort II received the following diagnoses: ALS/LMND 13, MMN 5, other diagnoses 4 (multifocal acquired demyelinating sensory and motor neuropathy (MADSAM), chronic inflammatory demyelinating polyneuropathy (CIDP), inclusion body myositis and multiple sclerosis) (Table 3). Of note, paraclinical findings considered to be supportive of MMN (GM1 antibodies, NCS conduction blocks) only offered limited group separation, with GM1 antibodies in up to 50\% of ALS/LMND patients and NCS conduction blocks in only up to $63 \%$ of MMN patients (Table 3).

\section{Cutoff value selection by ROC analysis}

Of 28 different US measurements in 12 different nerves/ nerve roots, 21 (75\%) showed significant group differences in cohort I with lower CSA/diameter in ALS/LMND than in MMN (Online Resource Table 3). ROC analyses in cohort I showed superior discrimination for US than for NCS between ALS/LMND and MMN (Fig. 1, NCS
CMAP, AUC (mean (stdv.)), 0.60 (0.09); US CSA/diameter, $0.82(0.12), p<0.001)$. All other NCS parameters (sensory and motor CVs, F responses) had even worse AUC than CMAPs (data not shown). Cutoff values to be used in the subsequent clinically guided diagnostic US models were determined for each single nerve by the ROC analysis in cohort I. Cutoff values with a minimum specificity of $80 \%, 90 \%, 95 \%$ and $100 \%$ or the highest Youden-Index were selected and systematically compared for their diagnostic performance in clinically guided US examination protocols (for cutoff values, see Table 3, and not shown).

\section{Determination of most heavily affected nervous structure by clinical deficits and examination site selection process}

The selection process for the most heavily affected nerve/ cervical root as the basis for measurement site selection in a clinically guided US protocol is given in Table 1 . In cohort I, C7-cervical root or radial nerve-dependent muscle functions were significantly more often primarily affected in MMN patients (50\% vs. $10 \%$ and $62 \%$ vs. $15 \%$, respectively, $p<0.05$ for both). In both cohorts, there was a trend for leg nerves to be more often primarily affected in ALS/LMND than in MMN patients ( $p>0.05$ for all).

The diagnostic potential of a clinically guided US examination protocol relies on a correlation between clinical deficits and US measurement values. Such a correlation was found for MMN patients only $(r(38)=-0.38, p=0.009$, all nerves, clinical deficits assessed by MRC-SS, correlated with $z$-transformed US values). This correlation was stronger if only the clinically most affected nerve of each patient was taken $(r(6)=-0.65, p=0.042)$, supporting the rationale of choosing the clinically most affected nerve as the site of US examination.

As to be expected, in many cases clinical deficits did not unequivocally identify one nervous structure. Single muscle testing in an individual patient often revealed muscle functions of two different nerves as being affected to the same extent. In case of distal muscle functions deficits could be attributed to consecutive nerves (e.g., sciatic vs. peroneal nerve for dorsal foot extension or $\mathrm{C} 7$ root vs. radial nerve for elbow extension).

Thus, different selection strategies can be chosen for the nervous structure to be examined by US based on individual clinical deficit distribution and were systematically compared for their diagnostic performance (for selection flow diagram and resulting models no. 1-8, see Fig. 2, for nerves finally selected according to each model, see Online Resource Table 3). 


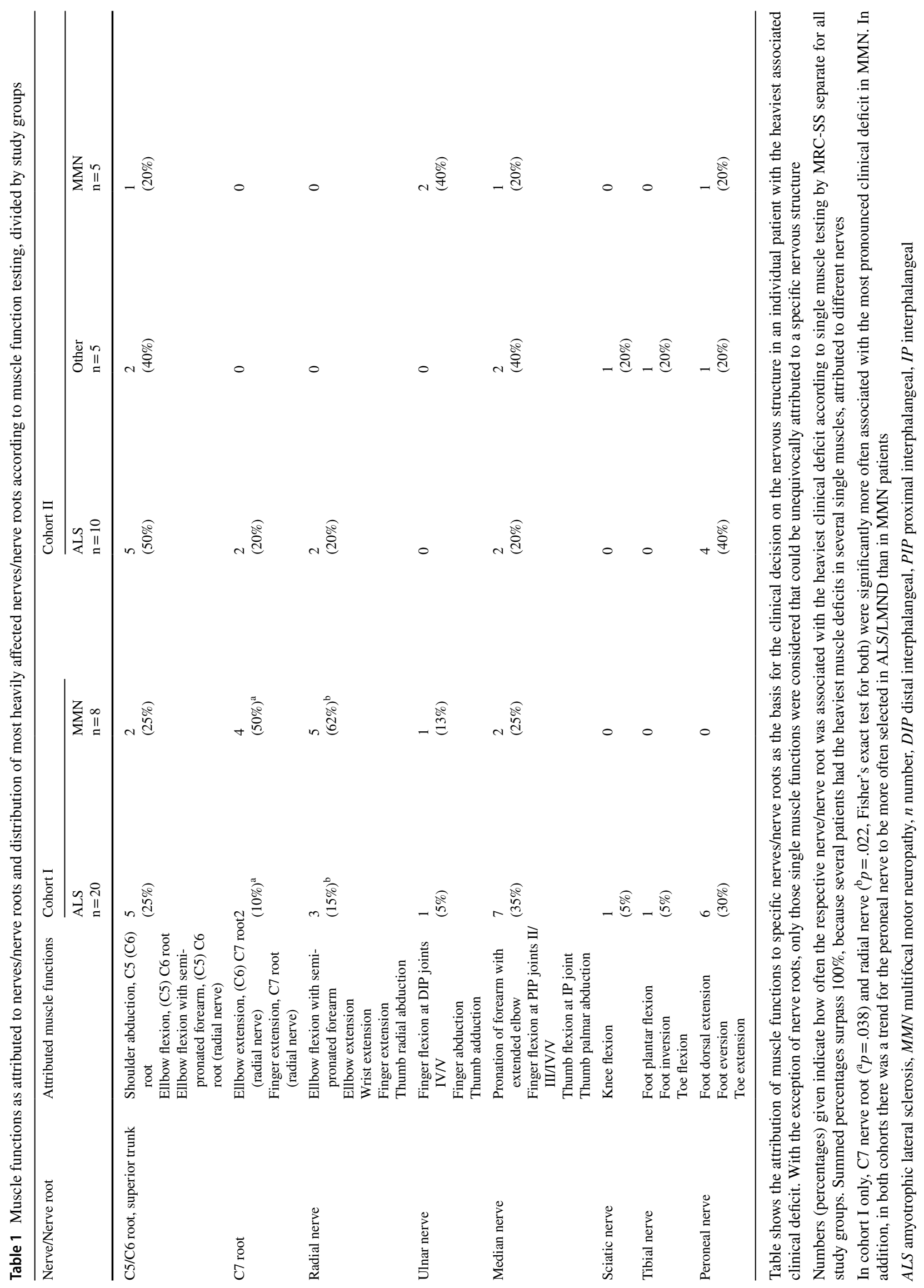


Table 2 Nerve-specific cutoff values (CSA \& diameter) with a minimum specificity of $95 \%$ as determined by group-based ROC analysis in cohort I

\begin{tabular}{lll}
\hline CSA & ROC AUC $(95 \%$ CI $)$ & $\begin{array}{l}\text { Cutoff } \\
\text { value } 95 \% \\
\text { specificity } \\
\text { for MMN } \\
\left(\mathrm{mm}^{2}\right)\end{array}$ \\
\hline Radial nerve upper arm & $1.00(1.00-1.00)$ & $\geq 4.50$ \\
Ulnar nerve upper arm & $0.86(0.70-1.00)$ & $\geq 6.68$ \\
Ulnar nerve forearm & $0.85(0.70-1.00)$ & $\geq 5.63$ \\
Median nerve upper arm & $0.83(0.65-1.00)$ & $\geq 9.33$ \\
Median nerve forearm & $0.83(0.61-1.00)$ & $\geq 7.12$ \\
Tibial nerve lower leg & $0.73(0.54-0.93)$ & $\geq 9.37$ \\
Sciatic nerve upper leg & $0.71(0.48-0.95)$ & $\geq 50.38$ \\
Peroneal nerve upper leg & $0.40(0.10-0.70)$ & $\geq 7.60$ \\
\hline Diameter & ROC AUC $(95 \%$ CI) & Cutoff \\
& & value $95 \%$ \\
specificity \\
for MMN \\
(mm)
\end{tabular}

Table shows cutoff values for CSA and diameter US measurement values with a minimum specificity of $95 \%$ in group ROC analysis in cohort I. These cutoff values were applied in the clinically guided individualized US protocols according to the decision flow diagram in Fig. 2. This procedure resulted in the diagnostic accuracies as given in Fig. 3. AUCs with a lower border of the $95 \%$ confidence interval of $\leq 0.50$ were not significant $(p \geq .50)$.

US ultrasound, CSA cross-sectional area, ROC receiver operating curve, $A U C$ area under the curve, 95\% CI 95\% confidence interval

\section{Diagnostic performance of clinically guided US protocols in comparison with clinically unguided former protocols and random examination site selection}

Starting with a minimum specificity of $95 \%$ of the cutoff value selected by group ROC analysis in cohort I, up to $100 \%$ specificity and sensitivity were achieved when applied to the strongest affected nerve (Fig. 3). Thus, these cutoff values were selected for further usage and comparison of diagnostic models (Table 2).

The accuracy of the clinically guided models based on the different selection strategies (Fig. 2, models 1-8) was compared to models based on clinically unguided random measurement value selection (Fig. 3, models $9 \& 10$ ) and to formerly published rigid examination protocols including 8 and 10 US values (Fig. 3, models $11 \& 12$ ), respectively $[2,4]$.

Overall, clinically guided US protocols, based on the clinically most affected nerve only, had superior diagnostic accuracy in both cohorts (Fig. 3, models 1-8), no matter whether compared to former rigid examination protocols or to models with random nerve selection. Among the clinically guided models, the ones including nerve root measurement sites (Fig. 3, models 2, 4, $6 \& 8$ ) had a tendency to a lower diagnostic accuracy than those with measurement values form peripheral nerves only (Fig. 3, models 1, 3, 5 \& 7). Inor exclusion of diameter measurements (Fig. 3, models $1 \&$ 2 vs. models $5 \& 7$ ) had no effect on accuracy, as well as the preference of proximal or distal measurement points in the case of consecutive nerves (Fig. 3, models $1 \& 5$ vs. models $3 \& 7$ ). The models with the highest diagnostic accuracy (Fig. 3, models 1,3,5 \& 7) reached 100\% correct classification rate for MMN in both cohorts, if patients with other diagnoses than ALS/LMND or MMN were excluded from the validation cohort II. If patients with other diagnoses were included, the specificity of these models dropped to $87 \%$, due to the misclassification of two subjects as MMN. These two subjects both had the final diagnosis of other acquired inflammatory demyelinating neuropathies (MADSAM or CIDP), in both cases due to a stronger sensory involvement than recognized during initial clinical evaluation at study inclusion.

\section{Discussion}

All diagnostic US models suggested so far for different neuropathies and/or ALS/LMND rely on rigid examination protocols that are not individualized according to the distribution of clinical deficits of the respective patient. Such rigid protocols do not consider the often uneven and in many cases even focused distribution of clinical deficits, even though US of nerval structures in an individual patient's clinical focus can be expected to be crucial for the highest diagnostic contrast. If such rigid examination protocols are too condensed and consequently risk leaving out the clinical focus, they could generate false-negative results and lower sensitivity. If such rigid examination protocols are extensive or even comprehensive for all accessible nerval structures, this can impede broad clinical implementation and generate false-positive results and lower specificity due to repetitive testing and due to the inclusion of technically challenging anatomic sites. To our knowledge, this is the first US study in both neuropathies and 


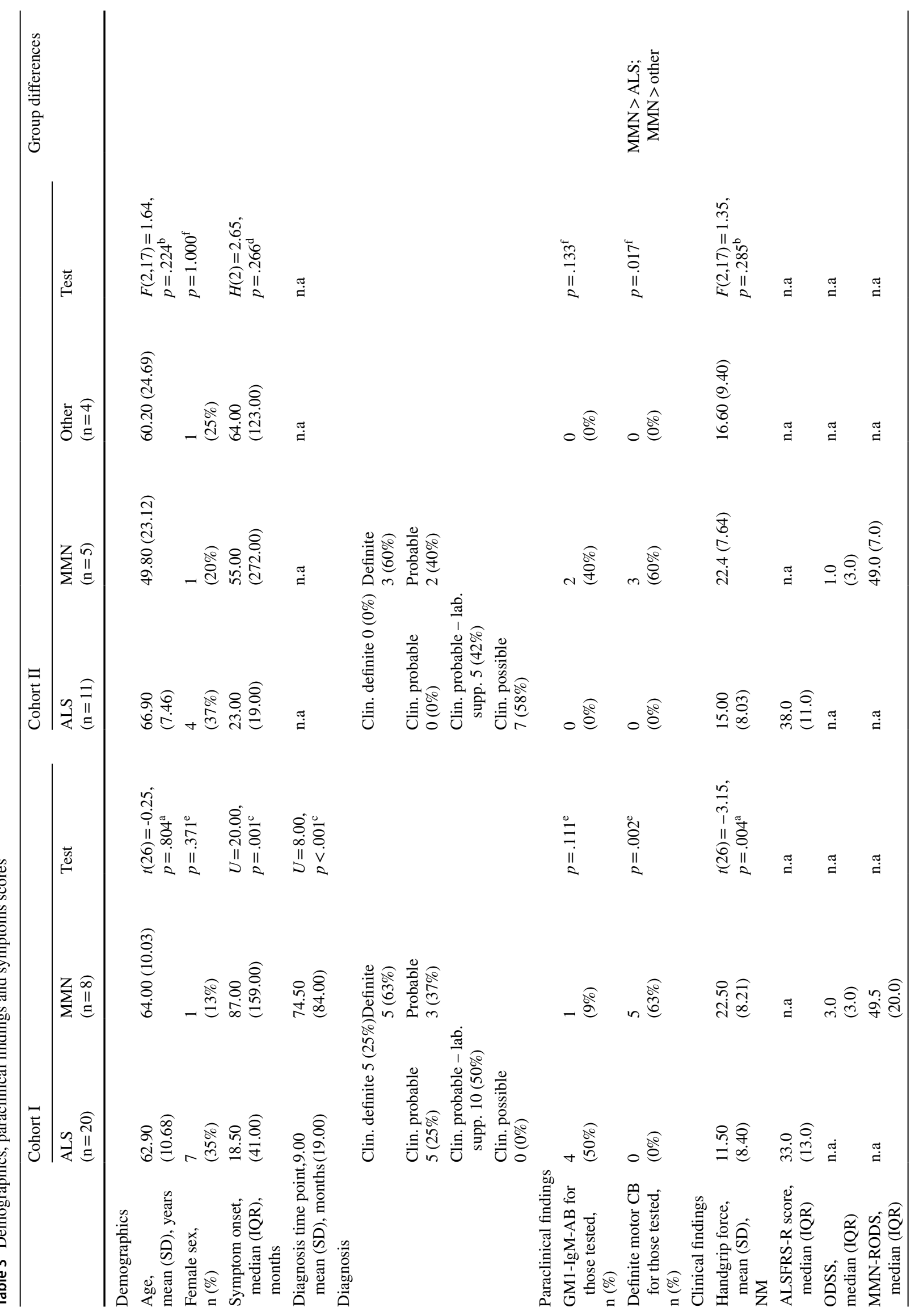




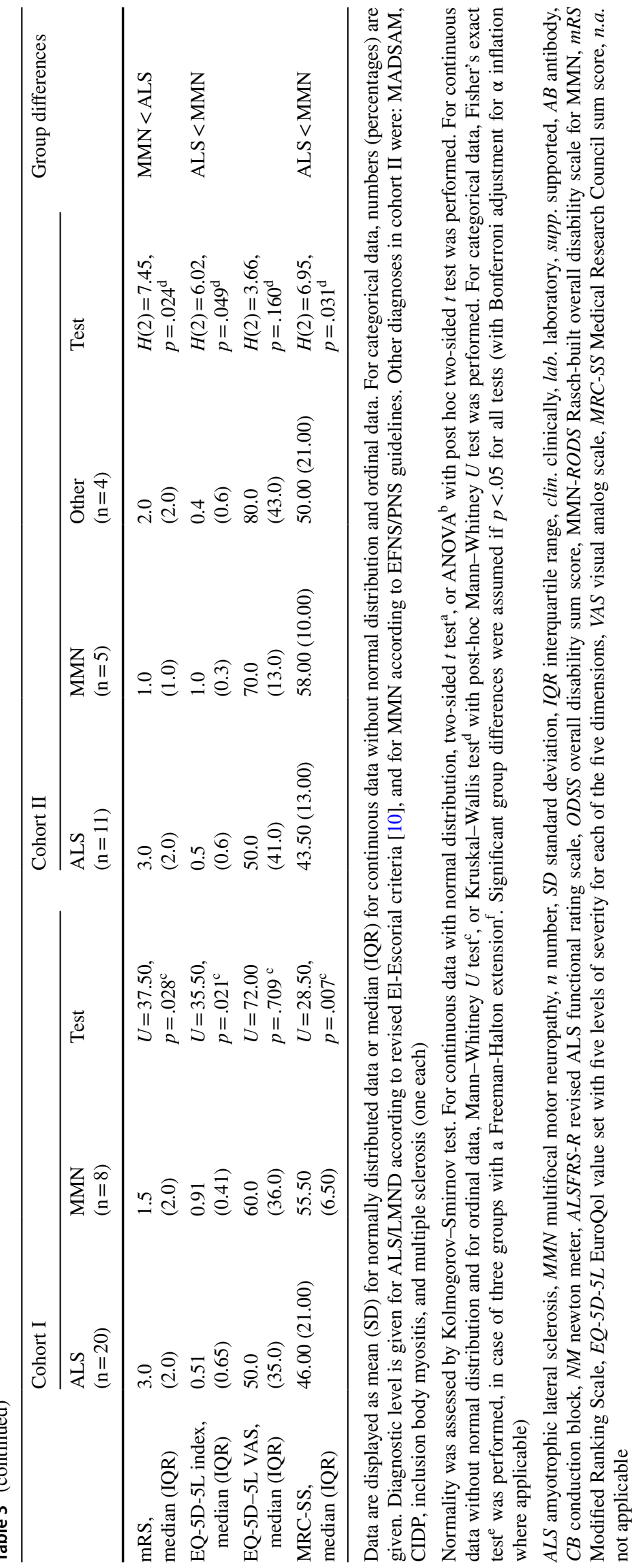




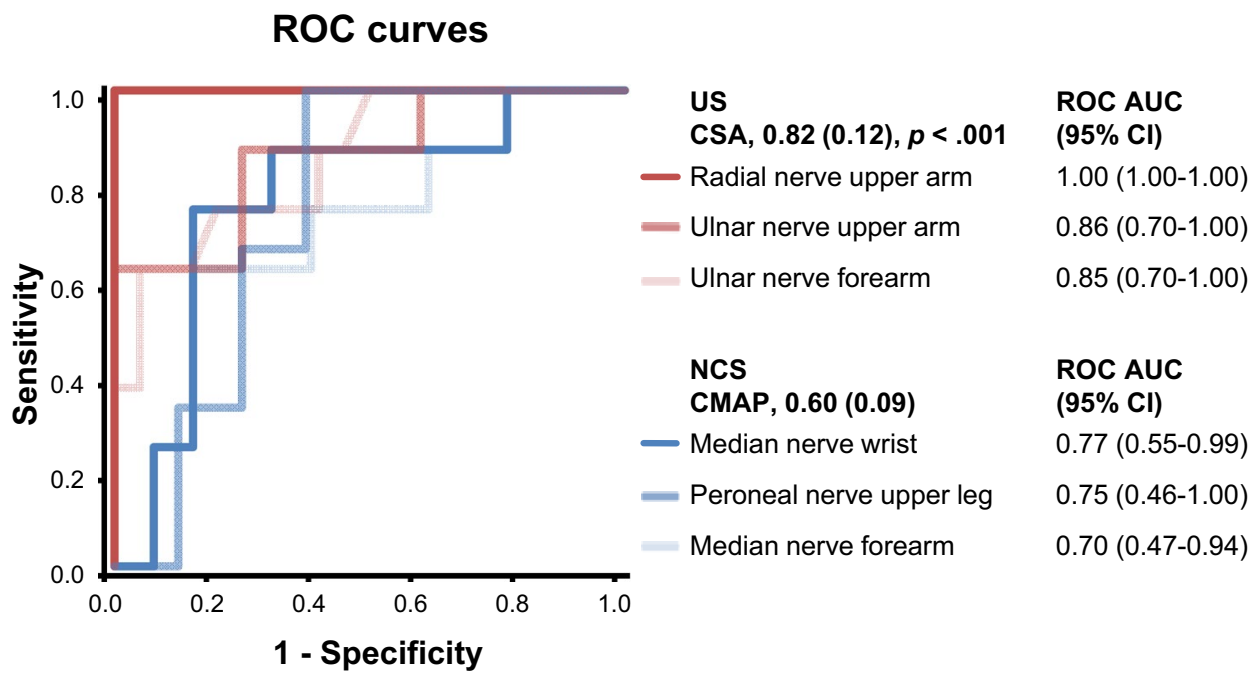

Fig. 1 ROC curves of best ultrasound (US) and nerve conduction studies (NCS) measurement values ROC curves of the three best US measurement values (as defined by highest AUC) as considered in the models with the best diagnostic performance (models no. 5 and 7, Fig. 3) are shown (red curves). In comparison, the three best values from NCS (all CMAPs, blue curves) had lower AUCs. AUCs with a lower border of the $95 \%$ confidence interval of $\leq 0.50$ were not sig-

ALS/LMND to abandon rigid examination protocols in favor for a clinically guided flexible examination protocol.

The results support both hypotheses that were the rationale of the current study:

Not only offered the clinically guided approach higher diagnostic accuracy in comparison to the rigid examination protocols formerly published by two independent research groups [2,4], reaching CCRs of up to $100 \%$ instead of $87 \%$ or $81 \%$ in cohort II (Fig. 3), but also was it more time-efficient. The inclusion of only nerve upstream of the most heavily affected muscle functions sufficed, instead of the 8 or 10 values to be measured for the former rigid examination protocols.

This increase in diagnostic accuracy of the clinically guided US protocols appears to be driven by the correlation of US changes and clinical deficits in $\mathrm{MMN}$ patients, whereas no such correlation could be found in ALS/LMND patients in our study group. This correlation in MMN patients is stronger in the clinically most affected nerve than in all nerves, further supporting the rationale to select the clinically most affected nerve for US examination.

Of the four models with equal and superior diagnostic accuracy (Fig. 3, models 1, 3, $5 \&$ 7), the most convenient model (Fig. 3, model 7) could be suggested for routine diagnostic application; Peripheral nerves are mostly more easily accessible by US than proximal ones (Fig. 3, model 5), and CSA are more conventionally used than diameter measurements (Fig. 3, models $1 \& 3$ ). Thus, a workflow nificant $(p \geq .50)$. Other classes of NCS values than CMAPs had even lower AUCs (not shown). Mean AUC of all US values (0.82 (0.12)) was significantly higher than mean AUC $(0.60(0.09))$ of all CMAP values at the best group of NCS values $(p<.001$; two-sided $t$-test). Abbreviations: $U S$ ultrasound, NCS nerve conduction studies, $C S A$ cross-sectional area, $R O C$ receiver operating curve, $A U C$ area under the curve, $95 \%$ CI 95\% confidence interval

for practical clinical application of model 7 could be: 1 . Decide on the clinically most affected nerve by single muscle function testing according to MRC-SS. 2. If there are two different nerves clinically equally affected, decide for the one easier to examine by US. If there are consecutive nerves responsible for the respective clinical deficit, decide for the more distal nerve. 3. Measure CSA values in that one nerve only and score the results according to the cutoff values given in Table 2.

In addition, several sensitivity analyses support the robustness of this approach: The same high accuracy was obtained whether or not diameter measurements in addition to CSA were considered (Fig. 3, models $1 \& 3$ vs. models 5 $\& 7$ ), or whether proximal or distal consecutive nerves were considered (Fig. 3, models $1 \& 5$ vs. models $3 \& 7$ ). The usefulness of a clinically guided measurement site selection was further supported by the inferiority of models based on random selection (Fig. 3, models $9 \& 10$, CCRs in cohort II: $66 \%$ and $79 \%$, respectively).

The current results are consistent with results of former descriptive or diagnostic studies on ALS/LMND and/or MMN: as to be expected based on known differences in clinical presentation $[18,19]$, the clinically most affected muscle functions were more often caudal in ALS/LMND than in MMN, such as foot or toe extension. As in former diagnostic US and NCS studies on the differentiation of ALS/LMND from MMN, ROC analyses showed a lower discriminatory power for NCS than for US (Fig. 1) [4]. 


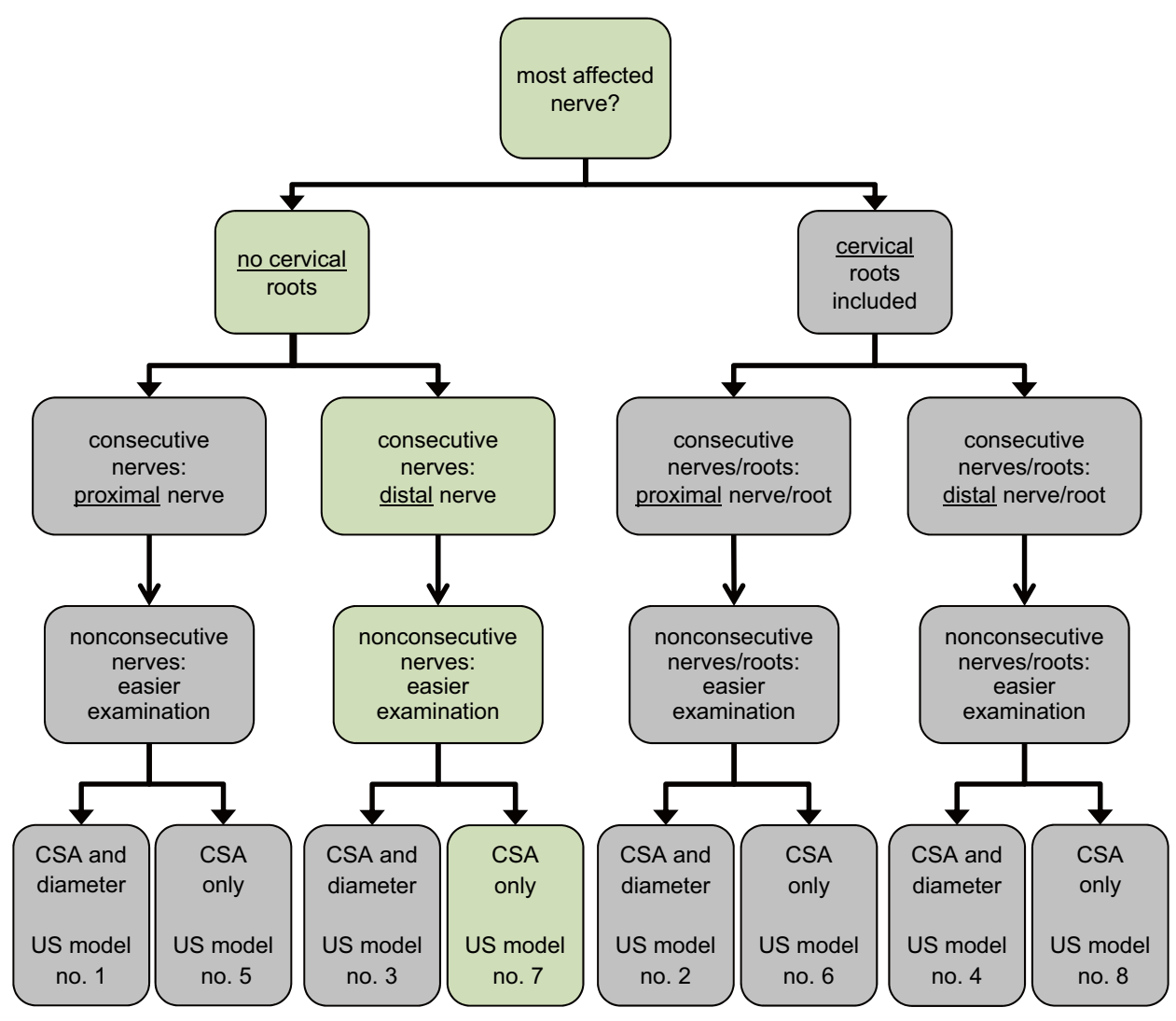

Fig. 2 Flow chart of different single nerve/nerve root selection strategies compared. Flowchart shows the different strategies assessed for the determination of the nerve/nerve root to be included into an individualized US measurement protocol based on the results of clinical testing of motor deficits in an individual patient. The model suggested for diagnostic application is shown in shaded green (model no. 7). This model is given preference because models without cervical roots had slightly better diagnostic performance (see Fig. 3) and distal nervous structures and CSA values were considered to be more easily measurable by US than proximal nervous structures and diameter measurements. However, diagnostic performance was stable or only slightly lower (models with cervical roots considered, see Fig. 3), independent of the specific measurement site selection strategy

Concerning the diagnostic potential of US measurements from cervical nerve roots in the contrast between ALS/LMND and MMN, former studies have heterogeneous results: Two former studies from independent study groups found a lower diagnostic potential of cervical nerve roots than peripheral nerves in the differentiation of ALS/LMND from MMN [4], or in the differentiation of ALS subtypes [20]. However, results of another study on the broader question of the differentiation of sensorimotor chronic inflammatory neuropathies from other forms of acquired neuropathies advise the cautious interpretation of the diagnostic potential of nerve roots [21]: The latter study achieved a similarly high diagnostic accuracy as the current study and included cervical roots in the measurement protocol. The diagnostic strategy differed substantially from the current one: The applied. The following models were compared: Models with (models no. $1,3,5 \& 7$ ) or without (models no. $2,4,6 \& 8$ ) consideration of cervical roots; Models with the consideration of the more proximal (models no. 1, 2, 5 \& 6) or more distal (models no. 3, 4, 7 \& 8) nerve/ nerve root in the case of the most pronounced deficit downstream to consecutive nervous structures. If two nonconsecutive nervous structures were affected to the same extent, the following was applied (to all models no. 1-8): selection of the nerve/nerve root easier to examine based on the following rules: (in order of increasing difficulty): (1) arm < leg < neck; (2) ulnar nerve $<$ median nerve < radial nerve; (3) peroneal nerve $<$ tibial nerve; (4) $\mathrm{C} 5$ root $<\mathrm{C} 6$ root $<\mathrm{C} 7$ root; (5) superior trunk $<\mathrm{C} 7$ root. Abbreviations: CSA cross-sectional area, no. number, $U S$ ultrasound

study was based on a rigid examination protocol including bilateral measurements from median nerves and cervical roots and relied on very rigid cutoff values of $99 \%$ specificity. The reason for these mixed results on the role of cervical roots remains speculative and warrants further investigation: Contributors could be differences in measurement protocol and strategies for cutoff value selection, an increased measurement error due to the technically more challenging examination of nerve roots compared to peripheral nerves, or differences of the individual distribution of morphologic changes in relatively small study cohorts.

In conclusion, the results of the current study suggest that a clinically guided US examination protocol, based on the assessment of the clinically strongest affected nerve only, could be both accurate and efficient in the differentiation 


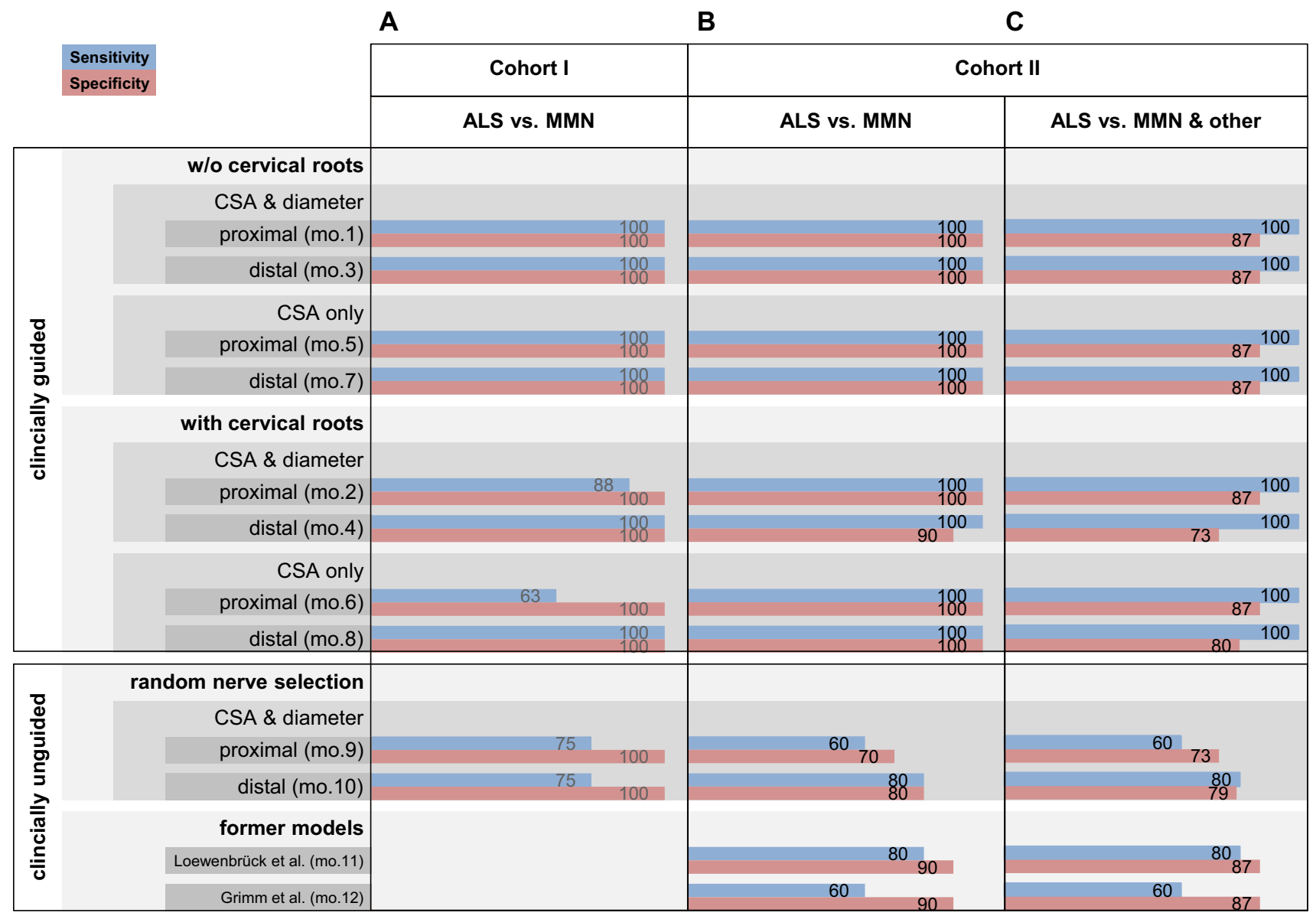

Fig. 3 Diagnostic performance of clinically guided US examination protocols based on different selection strategies in comparison to clinically unguided protocols and random examination site selection. Figure shows diagnostic performance as expressed by sensitivity and specificity of all eight different strategies (models no. 1-8) for a clinically guided selection of a single nerve/nerve root to be examined by ultrasound, and compares it with formerly published models with rigid clinically unguided examination protocols that are based on the assessment of 8 or 10 different measurement values (models no. $11 \& 12)[4,17]$. Additionally, the performance of all models is compared to random examination site selection (models no. 9 \& 10). It is indicated whether or not cervical roots were considered (models no. $2,4,6 \& 8$ vs. models no. $1,3,5$ \& 7), and whether or not diameter values were considered in addition to CSA values (models no. 1,2,3 $\& 4$ vs. models no. 5,6,7 \& 8). For details concerning the different strategies to select the examination site in the clinically guided protocols see Fig. 1. Clinically guided models no. 1-8 and models based

of ALS/LMND from MMN. In addition, implementation is technically relatively simple, since the acquisition of easyto-obtain CSA measurements from distal nerves obtained the same or higher diagnostic accuracy than other measurement site selection strategies compared.

Our study has several limitations. First, it was monocenter with a restricted number of participants. Even though patients were transferred from all district hospitals in the City of Dresden (> 530,000 inhabitants) and from nearby on random examination site selection (models no. $9 \& 10$ ) considered the cutoffs with a minimum specificity of $95 \%$ as derived from the group ROC analysis in cohort I (for cutoff values, see Table 2). In the formerly published models (models no. $11 \& 12$ ) the respective model-specific cutoff values were applied as published before $[4,17]$. A. shows the diagnostic performance of all models when applied to study cohort I. B. shows the diagnostic performance when applied to cohort II and patients with final diagnoses other than ALS/LMND or MNN were excluded. C. shows the diagnostic performance when patients with final diagnoses other than ALS/LMND or MMN were not excluded from analysis. If patients with other diagnoses were not excluded from cohort II, two of those were classified as MMN. These two patients had other chronic inflammatory neuropathies as final diagnoses because of a more important sensory involvement than initially considered at study inclusion. CSA cross-sectional area, mo. model, ALS amyotrophic lateral sclerosis, MMN multifocal motor neuropathy, no. number

regions of Eastern Saxony (> 1,000,000 inhabitants), only 20 patients could be recruited for cohort II. Thus, the reported diagnostic accuracy has to be regarded with caution. Results of the current study need to be reconfirmed in larger cohorts and by independent examiners.

This and other studies point out the importance to incorporate US into comprehensive and multimodal diagnostic workflows rather than to employ US as a separate complementary method. Whereas in the current study the 
distribution of clinical deficits was the premise for the US examination approach, in other studies NCS results were part of a defined multimodal diagnostic workflow and premise for a specific subsequent US examination [9, 22]. The promising potential of such defined sequential and multimodal diagnostic workflows should be further explored in future studies.

\section{Clinical implications}

In the differentiation of ALS/LMND from MMN, US examination guided by the distribution of clinical deficits in an individual patient offers higher diagnostic accuracy and is more efficient in direct comparison to clinically unguided examination strategies. Whether this also applies for nerve US examination in other diagnostic questions beyond the current one warrants further exploration.

Acknowledgements Open Access funding enabled and organized by Projekt DEAL. The authors would like to express their thankfulness to the participating study subjects, especially those who are highly handicapped and still participated. We would also like to thank Martin Pette for his help in electrophysiology diagnostics, Jochen Machetanz for referral of study participants and critical revision of the manuscript, and Jessica Drebes and Christiane Lehmann, technical assistants very supportive of our study.

Author contributions Dr. Loewenbrück was responsible for study concept and design, acquisition of data, statistical analyses and interpretation of data, and drafting the manuscript. Dr. Werner and Dr. Günther participated in study concept and design, acquisition of data, statistical analyses and interpretation of data, and drafting the manuscript. Dr. Klingenberger and Dr. Dittrich took part in acquisition of data, statistical analyses and interpretation of data. Dr. Reichmann took part in data interpretation and drafting of the manuscript. Dr. Hermann and Dr. Storch were responsible for study concept and design, acquisition of data, interpretation of data, and drafting of the manuscript. All authors critically revised the manuscript and approved its final version.

Funding Open Access funding enabled and organized by Projekt DEAL. This study was funded by the Bundesministerium für Bildung und Forschung (Federal Ministry for Education and Research Germany; AZ: 03WKBH2F) to A.S., and an unrestricted research grant from the Roland-Ernst Foundation to K.L. and A.S. A.H. is supported by the "Hermann und Lilly Schilling-Stiftung für medizinische Forschung im Stifterverband". R.G. was supported by niemALS aufgeben e.V. and by a family of an ALS patient. The funders had no role in the design and conduct of the study; collection, management, analysis, and interpretation of the data; preparation, review, or approval of the manuscript; and decision to submit the manuscript for publication.

Availability of data and material Data/Materials are made available in this publication and in the supplementary materials. Additional data/ materials are available from the authors upon request.

\section{Compliance with ethical standards}

Conflicts of interest The authors report no conflict of interest.
Ethics approval The study was approved by the responsible Ethics Review Committee at the Technische Universität Dresden, Germany. All study participants provided written informed consent prior to study inclusion. The study was conducted in accordance with the ethical standards laid down in the 1964 Declaration of Helsinki and its later amendments.

Open Access This article is licensed under a Creative Commons Attribution 4.0 International License, which permits use, sharing, adaptation, distribution and reproduction in any medium or format, as long as you give appropriate credit to the original author(s) and the source, provide a link to the Creative Commons licence, and indicate if changes were made. The images or other third party material in this article are included in the article's Creative Commons licence, unless indicated otherwise in a credit line to the material. If material is not included in the article's Creative Commons licence and your intended use is not permitted by statutory regulation or exceeds the permitted use, you will need to obtain permission directly from the copyright holder. To view a copy of this licence, visit http://creativecommons.org/licenses/by/4.0/.

\section{References}

1. van Schaik IN et al (2005) Intravenous immunoglobulin for multifocal motor neuropathy. Cochrane Database System Rev. https ://doi.org/10.1002/14651858.CD004429.pub2

2. Grimm A et al (2015a) Nerve ultrasound for differentiation between amyotrophic lateral sclerosis and multifocal motor neuropathy. J Neurol 262(4):870-880

3. Jongbloed BA et al (2016) Comparative study of peripheral nerve Mri and ultrasound in multifocal motor neuropathy and amyotrophic lateral sclerosis. Muscle Nerve 54(6):1133-1135

4. Loewenbrück KF et al (2016) Nerve ultrasound in the differentiation of multifocal motor neuropathy (MMN) and amyotrophic lateral sclerosis with predominant lower motor neuron disease (ALS/LMND). J Neurol 263(1):35-44

5. Cartwright MS et al (2011) Peripheral nerve and muscle ultrasound in amyotrophic lateral sclerosis. Muscle Nerve 44(3):346-351

6. Rattay TW et al (2017) Nerve ultrasound as follow-up tool in treated multifocal motor neuropathy. Eur J Neurol 24(9):1125-1134

7. Schreiber $S$ et al (2014) Peripheral nerve ultrasound in ALS phenotypes. Muscle Nerve. https://doi.org/10.1002/mus.24431

8. Schreiber $\mathrm{S}$ et al (2018) Peripheral nerve atrophy together with higher cerebrospinal fluid progranulin indicate axonal damage in amyotrophic lateral sclerosis. Muscle Nerve 57(2):273-278

9. Herraets IJT et al (2020) Nerve ultrasound improves detection of treatment-responsive chronic inflammatory neuropathies. Neurology 94(14):e1470-e1479

10. Brooks BR et al (2000) El Escorial revisited: revised criteria for the diagnosis of amyotrophic lateral sclerosis. Amyotroph Lateral Scler Other Motor Neuron Disord 1(5):293-299

11. van Schaik IN et al (2006) European Federation of Neurological Societies/Peripheral Nerve Society guideline on management of multifocal motor neuropathy. Eur J Neurol 13(8):802-808

12. Kleyweg RP, van der Meche FG, Schmitz PI (1991) Interobserver agreement in the assessment of muscle strength and functional abilities in Guillain-Barre syndrome. Muscle Nerve 14(11):1103-1109

13. Merkies IS, Lauria G (2006) 131st ENMC international workshop: selection of outcome measures for peripheral neuropathy clinical 
trials 10-12 December 2004, Naarden The Netherlands. Neuromuscul Disord 16(2):149-156

14. Vanhoutte EK, Faber CG, Merkies IS (2013) 196th ENMC international workshop: outcome measures in inflammatory peripheral neuropathies 8-10 February 2013, Naarden The Netherlands. Neuromusc Disord 23(11):924-933

15. Cedarbaum JM et al (1999) The ALSFRS-R: a revised ALS functional rating scale that incorporates assessments of respiratory function. BDNF ALS Study Group (Phase III). J Neurol Sci 169(1-2):13-21

16. Zaidman CM, Al-Lozi M, Pestronk A (2009) Peripheral nerve size in normals and patients with polyneuropathy: an ultrasound study. Muscle Nerve 40(6):960-966

17. Grimm A et al (2015b) Nerve ultrasound for differentiation between amyotrophic lateral sclerosis and multifocal motor neuropathy. J Neurol. https://doi.org/10.1007/s00415-015-7648-0
18. Delmont E et al (2006) Multifocal motor neuropathy with and without conduction block: a single entity? Neurology 67(4):592-596

19. Ravits J, Paul P, Jorg C (2007) Focality of upper and lower motor neuron degeneration at the clinical onset of ALS. Neurology 68(19):1571-1575

20. Nodera $\mathrm{H}$ et al (2014) Thinning of cervical nerve roots and peripheral nerves in ALS as measured by sonography. Clin Neurophysiol 125(9):1906-1911

21. Goedee HS et al (2017) Diagnostic value of sonography in treatment-naive chronic inflammatory neuropathies. Neurology 88(2):143-151

22. Loewenbrück KF et al (2018) Practically applicable nerve ultrasound models for the diagnosis of axonal and demyelinating hereditary motor and sensory neuropathies (HMSN). J Neurol 265(1):165-177 